\title{
Treatment of alopecia areata: an Australian expert
}

\author{
consensus statement
}

Short running title: Alopecia areata Australian consensus statement

Key words: totalis, universalis, azathioprine, cyclosporine, methotrexate

Authors: William C Cranwell ${ }^{1}$, Vivien Lai ${ }^{1,2}$, Louise Photiou ${ }^{1}$, Nekma Meah $^{1}$, Dmitri

Wall $^{1}$, Deepani Rathnayake ${ }^{1}$, Shobha Joseph ${ }^{1}$, Vijaya Chitreddy $^{1}$, Shyamalar

Gunatheesan $^{1}$, Kamaldeep Sindhu ${ }^{3}$, Pooja Sharma ${ }^{1}$, Jack Green ${ }^{4,5,6}$, Samantha

Eisman $^{1}$, Leona Yip ${ }^{4,7}$, Leslie Jones ${ }^{1,8,9}$, Rod Sinclair ${ }^{1,7,8}$

1. Sinclair Dermatology, Victoria, Australia

2. Department of Medicine, Nursing and Health Sciences, Monash University, Victoria, Australia

3. North Western Dermatology, Victoria, Australia

4. Department of Dermatology, St Vincent's Hospital, Melbourne, Victoria, Australia

5. Skin and Cancer Foundation Inc, Melbourne, Victoria, Australia

6. Western Dermatology, Werribee, Victoria, Australia

7. Australian National University Medical School, Canberra, Australian

Capital Territory, Australia

8. Epworth Dermatology, Victoria, Australia

9. Department of Medicine, University of Melbourne, Victoria, Australia

Corresponding Author: Rodney Sinclair MBBS, MD, FACD

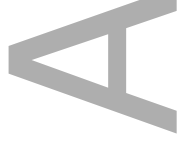

Professor of Dermatology

University of Melbourne and Sinclair Dermatology

2 Wellington Parade

This is the author manuscript accepted for publication and has undergone full peer review but has not been through the copyediting, typesetting, pagination and proofreading process, which may lead to differences between this version and the Version of Record. Please cite this article as doi: 10.1111/ajd.12941

This article is protected by copyright. All rights reserved 


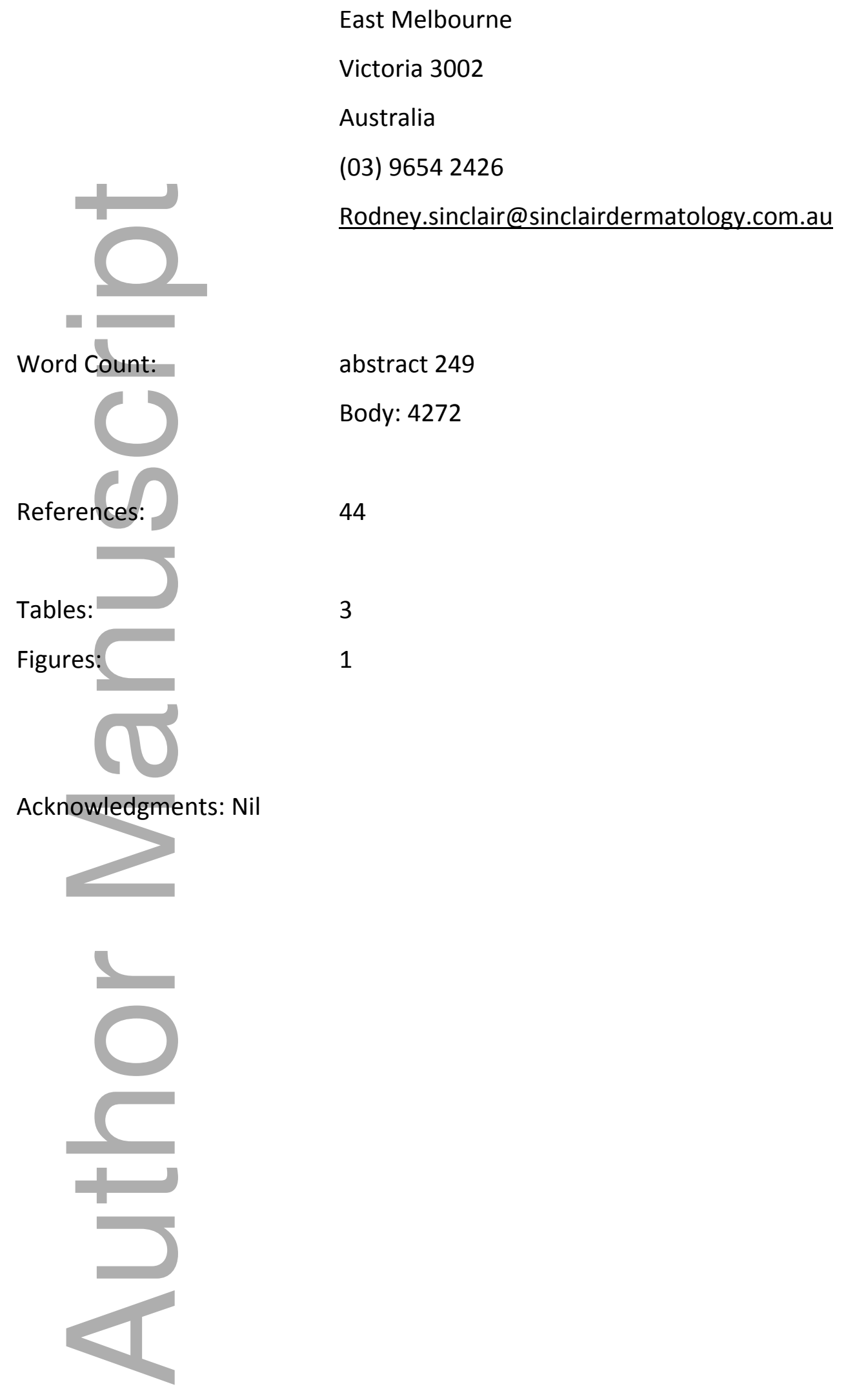

This article is protected by copyright. All rights reserved 
DR. WILLIAM CHARLES CRANWELL (Orcid ID : 0000-0001-6368-5738)

DR. VIVIEN WAI YUN LAI (Orcid ID : 0000-0003-4530-9864)

DR. LOUISE PHOTIOU (Orcid ID : 0000-0002-9441-3943)

Article type : Consensus Article

\section{Abstract}

Alopecia areata (AA) severity varies from a single small patch to complete loss of scalp hair, body hair, eyelashes and eyebrows. While $40 \%$ of all affected individuals only ever get one patch and will achieve a spontaneous complete durable remission within 6 months, $27 \%$ will develop additional patches but still achieve complete durable remission within 12 months and $33 \%$ will develop chronic AA. Without systemic treatment, $55 \%$ of individuals with chronic AA will have persistent multifocal relapsing and remitting disease, $30 \%$ will ultimately develop alopecia totalis (AT) and $15 \%$ will develop alopecia universalis (AU). The unpredictable course and psychological distress attributable to AA contributes to the illness associated with AA. Numerous topical, intralesional and systemic agents are currently used to treat $A A$, however there is a paucity of data evaluating their use, effectiveness and tolerability. Topical therapy, including topical glucocorticosteroids, topical minoxidil and topical immunotherapy, can be used in cases of limited disease. There are no universally agreed indications for initiating systemic treatment for AA. Possible indications for systemic treatment include rapid hair loss, extensive disease ( $\geq 50 \%$ hair loss), chronic $A A$, severe distress, or a combination of these factors. Currently available systemic treatments include glucocorticosteroids, methotrexate, ciclosporin, azathioprine, dapsone, mycophenolate mofetil, tacrolimus, and sulfasalazine. The optimal treatment algorithm has not yet been described. The purpose of this consensus statement is to outline a treatment algorithm for $A A$, including the indications for systemic treatment, appropriate choice of systemic treatment, satisfactory outcome measures and when to discontinue successful or unsuccessful treatment. 


\section{Learning Points}

- No systemic agents are currently approved for use by the Food and Drugs Administration or Therapeutic Goods Administration. There are no evidence-based Australian or International treatment guidelines for systemic therapy of AA.

- This consensus statement addresses the rationale for systemic treatment, the choice of systemic treatment, requirements for monitoring of systemic treatment, assessment of response to treatment, and appropriate cessation of therapy in AA.

\section{Introduction}

Alopecia areata (AA) is an immune-mediated disease that produces non-scarring hair loss. AA may occur as: an acute self-limiting disorder with 1-5 patches that resolve within 6-12 months, as a chronic disorder with multiple patches relapsing and remitting over many years, or as total hair loss of the scalp or universal loss of every terminal hair on the body. The estimated prevalence is 1 in 1000 people, with a lifetime risk of approximately $2 \%(1,2)$. The onset of AA typically occurs before age 40 , however late onset is well described $(2,3)$. Men and women appear to be equally affected and there is no known racial predisposition.

Some patients regrow spontaneously without medical intervention within 12 months (3). Many can be managed with topical or intralesional treatments alone. No systemic agents are currently approved for use by the Food and Drugs Administration or Therapeutic Goods Administration. There are no evidence-based Australian or International treatment guidelines for systemic therapy of AA.

The response of $A A$ to treatment is unpredictable. Even during a course of successful treatment, minor relapses can occur. It is not uncommon for a patient to develop a new lesion of AA on one part of the scalp while simultaneously experiencing regrowth in a recently treated patch of $A A$ on another part of the scalp.

Neither a 2008 Cochrane review, nor a 2018 systematic reviewidentified any systemic therapy for AA supported by level A evidence $(4,5)$. In the absence of level A evidence, an expert consensus statement can help guide management. This consensus statement will address the rationale for initiating topical and systemic treatment, the choice of systemic 
treatment, requirements for monitoring of systemic treatment, assessment of response to treatment, and appropriate cessation of therapy in AA.

\section{Methods}

A meeting of members of the Australasian Hair and Wool Research Society was convened at the $10^{\text {th }}$ World Congress for Hair Research in Kyoto in November 2017. Members of this meeting included Australian dermatologists with a sub-specialist expertise in alopecia, a research scientist with extensive experience in hair biology and disease and a dermatology clinical research fellow. Following a literature review, discussion was conducted at the Kyoto meeting and subsequently via correspondence. A consensus statement for the systemic treatment of AA was developed from these discussions and distributed to senior Australian Dermatologists for comment.

\section{Rationale for treatment}

In her landmark 1963 paper (Table 1), Ikeda described the natural history of AA among 1989 patients in Kyoto, Japan between 1947 and 1963 (3). Most patients did not receive active treatment. Only 302 patients received oral corticosteroids up to a maximum dose of prednisolone $5 \mathrm{mg}$ daily (or equivalent dose of dexamethasone). She showed that $40 \%$ of patients only ever develop a solitary patch of AA that regrows spontaneously within 6 months (3). Another $27 \%$ develop additional patches, but still achieve a complete and persistent remission at one year $(3,6)$. Patients with chronic AA, defined as AA that continues beyond one year, tend to develop additional areas of AA and have persistent hair loss for many years. Many never achieve complete remission. Among those with chronic AA, 45\% ultimately develop either alopecia totalis (AT) or universalis (AU) (30\% and 15\% respectively) (3). A longitudinal study in South Korea of 70 patients with AT or AU showed only $17 \%$ complete regrowth overall ( $20 \%$ and $15 \%$ respectively) (7). No hair regrowth was seen in $65 \%$ and $21 \%$ of cases of AU or AT respectively (7).

Possible indicators of poor prognosis at the time of initial presentation include AA onset before the age of 12 and in particular before the age of 6 years, disease duration of more than one year, development of multiple discrete patches, extensive hair loss involving $>50 \%$ This article is protected by copyright. All rights reserved 
of the scalp, ophiasis pattern of alopecia, progression to AT or AU, associated nail disease, associated Trisomy 21, associated atopy, and a positive family history for AA or other organ specific auto-immune disease $(6,8)$.

An increased prevalence of psychiatric comorbidity, particularly mood and anxiety disorders, is well established in patients with AA $(9,10)$. Significant psychological distress is particularly prevalent in those with severe or recalcitrant disease. Children and adolescents with AA experience high rates of depression and anxiety disorders (11). Parents of children affected by AA may also experience anxiety and mood disturbance. In view of the unpredictable course of disease and significant morbidity and distress, many patients seek treatment for $A A$.

The aim of treatment of $A A$ is to arrest disease progression and to reverse hair loss. While there is no current evidence that active treatment alters the natural history of $A A$, patients with multilocular AA generally develop lesions serially over a number of months. Patients rarely develop multiple discrete patches at the same time. There appears to be an accentuation of $A A$ severity with the risk of developing each new lesion of $A A$ increasing with disease severity.

While some patients who have recovered from an episode of acute AA, and many who are in complete remission following chronic $A A$, may experience a relapse of their $A A$ at some point in the future $(12,13)$ it is not known whether active treatment of acute AA prevents development of chronic AA. An analysis of 68 patients with chronic AA who received systemic treatment at a single large Melbourne alopecia clinic and who were followed for between 2 to 7 years found the rate of AT/AU was reduced to $17.6 \%$ (Table 2). The anticipated rate of AT/AU based on the lkeda paper was $45 \%$ (3). These results require further investigation, but suggest there is a logical rationale to treat chronic AA to reduce the risk of AT/AU. However, long term maintenance therapy to prevent relapse is currently not encouraged as the interval between episodes is unpredictable and may be years or even decades. Therefore the potential side effects of systemic therapy have thus far outweighed the potential benefit of treatment. 
Possible Indications for initiation of treatment

\section{Solitary Stable Patch of AA}

For many patients the presence of a single patch is cause for concern. Patients who experience significant mood change or anxiety that may impair social function (e.g. school avoidance) should be treated. Topical high potency corticosteroid in a child and intralesional corticosteroid injection repeated every 4-6 weeks in an adult are appropriate first line therapies.

Thirty five percent of individual solitary lesions that have persisted beyond 6 months will still resolve spontaneously by 12 months, while $65 \%$ will persist and evolve into chronic AA (3). All solitary lesions present longer than 6 months should be considered for topical or intralesional therapy and all lesions that have persisted for 12 months and which have failed a 6 month trial of topical or serial intralesional corticosteroid should be considered for topical immunotherapy or systemic treatment. Likewise, patients with a large lesion of AA, who cannot be easily managed with intralesional therapy due to the discomfort associated with the number of injections required, and patients who experience dermal atrophy following intralesional corticosteroid or side effects following topical steroid use, should also be considered for topical immunotherapy or systemic therapy.

The surface area of the average male scalp is approximately $630 \mathrm{~cm}^{2}$. AA affecting more than $15 \%-20 \%$ of the scalp $\left(95-130 \mathrm{~cm}^{2}\right)$ generally will require systemic therapy.

\section{Solitary Active Patch of AA}

An active patch of AA is characterised by a history of enlargement, a positive hair pull test at the border, and the presence of exclamation mark hairs or black dots within the patch. Activity within a patch of $A A$ is an indication for active treatment. 
Topical high potency corticosteroid in a child and intralesional corticosteroid injection repeated every 4-6 weeks in an adult are appropriate first line therapies in the presence of active disease.

\section{Multiple patches}

All patients with more than one patch should be considered for active treatment. Fifty-three percent of patients with AA develop additional patches over a number of months (3). Some may be unaware of the initial patch and only notice it when a second area appears. Among patients with multiple patches of AA, $65 \%$ will achieve complete remission within 12 months while $35 \%$ will progress to chronic disease (3).

Topical high potency corticosteroid in a child and intralesional corticosteroid injection repeated every 4-6 weeks in an adult are appropriate first line therapies. Topical immunotherapy is an alternate first line therapy in children. AA that fails to respond to a 6 month trial of topical or serial intralesional corticosteroid should be considered for topical immunotherapy or systemic therapy.

Patients with stable extensive disease (more than $95-130 \mathrm{~cm}^{2}$ ), who cannot be easily managed with intralesional therapy due to the discomfort associated with the number of injections required, should be considered for topical immunotherapy or systemic therapy. Patients who experience dermal atrophy following intralesional corticosteroid or cutaneous side effects following topical steroid use should be considered for topical immunotherapy or systemic therapy.

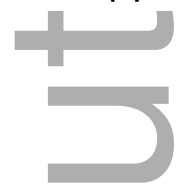

\section{Ophiasis alopecia}

There is no available data on the rate of spontaneous regrowth among patients who present with AA in an ophiasis pattern, however there is expert consensus that hair loss with an ophiasis pattern indicates a poorer prognosis and merits active treatment. 
Topical high potency corticosteroid in a child and intralesional corticosteroid injection repeated every 4-6 weeks in an adult are appropriate first line therapies. Topical immunotherapy is considered an alternate first line therapy in children. Due to large surface area of involvement, as described previously, these treatments may be intolerable or ineffective. Therefore, $A A$ that fails to respond to a 6-month trial of topical or serial intralesional corticosteroid should be considered for topical immunotherapy or systemic therapy.

Patients with stable extensive disease (more than $95-130 \mathrm{~cm}^{2}$ ), who cannot be easily managed with intralesional therapy due to the discomfort associated with the number of injections required should be considered for topical immunotherapy or systemic therapy. Patients who experience dermal atrophy following intralesional corticosteroid or cutaneous side effects following topical steroid use may also be considered for topical immunotherapy or systemic therapy.

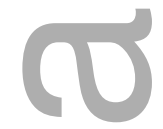

\section{Rapid progressive hair loss/Diffuse AA/ Extensive AA/ AT or AU}

Seventy percent of patients who develop AT/AU do so rapidly and within 4 months of disease onset, while $30 \%$ take longer than 30 months to develop AT/AU (3). Rapid progression of hair loss that is likely to result in $\geq 50 \%$ hair loss, AT or AU are indications for systemic therapy as topical and intralesional therapy are unlikely to arrest hair loss in these circumstances.

In a placebo controlled trial evaluating patients with $>50 \%$ hair loss due to $A A$, significant regrowth among patients in the placebo group was only seen in $7 \%(14)$.

\section{Systemic therapy in Children}


Children who develop AA before the age of 6 have a very high risk of developing chronic, persistent and extensive AA. In Ikeda's study there were 53 children below the age of 6 and no child regrew their hair spontaneously and was disease free at 12 months (3). All 53 children developed chronic AA.

Ikeda identified 83 children aged between 7 and 12 years at the time of initial presentation of their AA. Forty-seven had complete recovery of their hair within 3 years, while 36 went on to chronic persistent extensive disease. Half of these children then progressed rapidly to chronic persistent extensive disease, while the other 18 recovered initially and then relapsed and then developed chronic persistent extensive disease (3).

All children with AA should be considered for active treatment. Intralesional corticosteroid injection is generally not feasible without sedation and we believe the risks associated with sedation are not justified in the treatment of AA. Topical corticosteroid is therefore considered the most appropriate first line therapy in children with AA. Topical minoxidil and topical immunotherapy are alternate first line therapies in children. A combination of two or all three of these treatments is considered second line management. Systemic therapy is considered a third line therapy in children. In the absence of data confirming that systemic therapy reduces the risk of subsequent development of AT or AU, there is no consensus opinion on the use of systemic therapy in children.

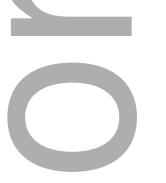

Other Associations with AA

The presence of associated nail pits is seen in up to $30 \%$ of patients with $A A(15,16)$. Nail pitting does not correlate with hair loss severity and nail pits may precede hair loss by many years. While commonly thought to indicate worse prognosis in AA, the presence of nail pits alone does not alter our treatment algorithm. Trachyonychia is an uncommon nail manifestation of AA, present in approximately 4\% of patients with AA in a review of 1095 patients $(15,17)$.

This article is protected by copyright. All rights reserved 
Ikeda identified a past or family history of atopy as an indicator of poor prognosis in AA. This has not been confirmed in subsequent studies $(18,19)$. The presence of a past or family history of atopy does not alter our treatment algorithm.

Ikeda identified 'endocrine-autonomic' disease as an indicator of poor prognosis in AA. She defined endocrine-autonomic disease as a personal history of diabetes mellitus, peptic ulcer, bilateral oophorectomy, hypertension, atherosclerosis, Basedow's disease and myxoedema, but without a personal history of atopy. This has not been confirmed in subsequent studies (19-21). The presence of a past or family history of endocrineautonomic disease does not alter our treatment algorithm.

AA is known to be associated with other organ specific auto-immune diseases such a Graves disease, vitiligo, pernicious anaemia, Coeliac disease and Type 1 Diabetes Mellitus (22). Genome-wide association studies in AA have identified a number of candidate genes common to these organ specific autoimmune diseases, suggesting common molecular pathways in their pathogenesis $(23,24)$. The presence of a past or family history of autoimmune disease does not alter our treatment algorithm.

Trisomy 21 (Down Syndrome) is associated with an increased prevalence (9\%) and severity of AA (25). It is estimated that $40 \%$ of patients with trisomy 21 will develop $A T / A U(25,26)$. Topical corticosteroids are considered the appropriate first line therapy in patients presenting with AA on a background of Trisomy 21. Topical minoxidil and topical immunotherapy are alternate first line therapies. In our experience, intralesional corticosteroids tend to be poorly tolerated due to pain and distress. Systemic corticosteroids may increase appetite and anecdotally, this can be very difficult for carers to manage. Patients with Trisomy 21 have an increased risk of developing acute lymphoblastic leukaemia and acute myeloid leukaemia (27). This is a relative contra-indication to systemic therapy in Trisomy 21 patients with AA.

\section{Diagnosis and baseline investigation}


AA is a clinical diagnosis based on visual inspection of the lesions and dermoscopy. In atypical cases a scalp biopsy may be helpful. While there are a number of known disease associations, there is insufficient data to recommend routine screening for concomitant autoimmune disease at the time of diagnosis of AA . Investigations for co-morbidities should be based on history and examination, taking into account any intended use of systemic therapy.

Conservative Management

For patients with limited stable disease that is inconspicuous or easily camouflaged, reassurance alone may be appropriate. Camouflage options include colour matched wool fibres ground into a powder that is dusted onto the hair, and dyes that are applied to the skin as a cream or aerosol spray to conceal the scalp. Wigs, top pieces and hair extensions can also be used.

\section{Topical therapy}

Patients with limited disease may seek active treatment in order to accelerate regrowth. Options include topical glucocorticosteroids, topical minoxidil and topical immunotherapy. Agents used for topical immunotherapy include diphencypropenone, dinitrochlorobenzene, squaric acid dibutyl ester and dithranol. A detailed discussion of the use of these agents is beyond the scope of this expert consensus statement.

\section{Intralesional glucocorticosteroids}

Intradermal or upper subcutaneous injection of long acting triamcinolone or betamethasone has been widely used to treat AA. Dilute concentration should be as effective as undiluted triamcinolone in some patients $(28,29)$. Individual patches of AA generally respond well to serial intralesional injection of corticosteroid repeated every 4-6 weeks. In a study that evaluated response to intralesional triamcinolone through dermoscopy, 60 of 70 patches of AA in 70 patients demonstrated regrowth of new vellus hair at 4 weeks (30). Possible side-effects include pain, dermal atrophy at the injection site 
and systemic absorption. A detailed discussion of the use of these agents is beyond the scope of this expert consensus statement.

3. Intramuscular and intravenous glucocorticosteroids

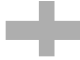

Long acting glucocorticosteroids can be administered intramuscularly or intravenously as an alternative to oral ingestion. While there has been some suggestion in the literature that intramuscular or intravenous injection has a superior side-effect profile compared to oral ingestion (31), there is no uniform consensus on this (32) and unless there is an contraindication to oral ingestion we favour oral corticosteroid over intramuscular or intravenous injection.

4. Systemic glucocorticosteroids

Up to 89 of patients respond to oral corticosteroids, while at least $11 \%$ are refractory to treatment even with high doses (33). Among those who respond, 50\% will relapse with dose reduction or soon after discontinuation of treatment. Long-term use of systemic glucocorticoids is not recommended due to associated side effects. For patients who are corticosteroid responsive, but also corticosteroid dependent, a switch to an appropriate steroid-sparing agent should be considered.

The initial dose of systemic glucocorticoid selected by the treating physician may vary depending on severity of $A A$ and treatment history. Dosing strategies include: commencing with a higher dose of oral prednisolone $(0.5-0.75 \mathrm{mg}$ per $\mathrm{kg}$ ) with subsequent tapering from 6 to 12 weeks; maintaining a static dose of prednisolone $(0.25 \mathrm{mg} / \mathrm{kg})$ for $6-12$ weeks; or commencing a lower dose $(0.1-0.2 \mathrm{mg} / \mathrm{kg})$ and increasing the dose over time according to response and tolerance. While most of us commence treatment with an initial dose of oral prednisolone of $0.5 \mathrm{mg} / \mathrm{kg}$, to be tapered down over 6-12 weeks, there is no expert consensus on the optimal use of oral prednisolone in AA. While a number of dermatologists favour pulsed oral corticosteroids, there is little evidence that this offers superior efficacy or safety [28]. A cross-sectional study of children with severe AA found 
that pulsed systemic corticosteroid therapy modified the initial course but did not generally influence the long-term outcome (34).

While we suspect that failure to achieve satisfactory hair regrowth with systemic corticosteroids predicts a lower response rate to systemic steroid-sparing anti-inflammatory agents, there are no randomised controlled trials (RCTs) that address this question specifically. Repeat courses of systemic glucocorticosteroids may be indicated for relapse following initial regrowth, however long-term maintenance therapy is not recommended. Subsequent courses should be initiated at a lower dose in patients known to respond to prednisolone.

Long-term use of glucocorticoid therapy is not recommended due to associated side effects. Short-term use should also be monitored carefully. The prevalence of side effects vary depending on the daily dose, duration of therapy, and patient comorbidities.

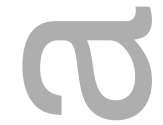

5. Steroid-sparing agents - Azathioprine, Methotrexate and Ciclosporin

There are no RCTs supporting the use of any second-line systemic agents in the treatment of AA. Agents used in open label retrospective case series or small prospective studies include azathioprine, methotrexate and ciclosporin. These agents should be used alone or in combination with prednisolone. They appear to be most effective when used as steroid sparing agents to prevent relapse of $A A$, rather than as monotherapy to initiate regrowth in AA.

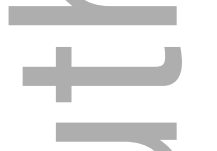

There is no expert consensus regarding the choice of a certain steroid-sparing agent over another and the decision to use one should therefore be based on clinical judgment and experience in the context of the patient's comorbidities, relevant past history and lifestyle factors.

There is no uniformly accepted way to judge the relative efficacy of these agents either, particularly in view of the fluctuating natural history of $A A$, the frequent use of multiple 
medications simultaneously and the relatively high rate of side-effects leading to dose reduction or cessation. In order to retrospectively assess the efficacy of these steroidsparing anti-inflammatory agents in our clinic, we evaluated the number of patients in a single large Melbourne hair loss clinic who continued taking the medication continuously for 12 months (Table 3). This is predicated on the basis that only patients who tolerated the medication and in whom both the patient and the treating doctor were satisfied it was working would continue to take the medication. Patients who ceased the medication due to complete remission prior to 12 months were considered to be responders.

Azathioprine is commonly started at a low dose $(0.5-1 \mathrm{mg} / \mathrm{kg}$ daily) to minimise the risk of $\mathrm{Gl}$ upset and gradually titrated every 4-6 weeks up to $2-3 \mathrm{mg} / \mathrm{kg}$ according to patient response and tolerance. About one third of patients continue to take concomitant prednisolone and most have serial injections of triamcinolone into areas of residual hair loss and disease activity (Table 3). All patients should have their serum TPMT measured prior to commencing therapy. Biochemical monitoring should be performed at least 4 times per year.

Methotrexate is commonly initiated at 5-10 mg once weekly and the dose gradually titrated every 4-6 weeks up to 20-30 mg according to patient response and tolerance. More than half the patients continue to take concomitant prednisolone and most have serial injections of triamcinolone into areas of residual hair loss and disease activity (Figure 1). Patients should take folic acid daily (except on the methotrexate day). Biochemical monitoring should be performed at least 4 times per year.

Ciclosporin A is commonly initiated at $2 \mathrm{mg} / \mathrm{kg}$ daily in 3 divided doses and the dose gradually titrated every 4-6 weeks up to a maximum of $5 \mathrm{mg} / \mathrm{kg}$ according to patient response and tolerance. Only a third of patients are able to cease prednisolone but most continue to have serial injections of triamcinolone into areas of residual hair loss and disease activity (Table 3). Patients should have their blood pressure monitored regularly and have urinalysis and biochemical monitoring at least 6 times per year. 
Minor relapses while on steroid-sparing therapy tend to be treated with the addition of intralesional triamcinolone or short-term glucocorticoids. Major relapses are generally managed by cessation of the steroid-sparing agent and substitution with an alternate steroid-sparing agent.

\section{Other systemic therapies}

Regrowth of hair during treatment with tofacitinib, a small molecule Janus kinase $1 / 3$ inhibitor, has been described in numerous case series (35-39). In a retrospective study of 90 patients with severe AA who received tofacitinib (5 to $10 \mathrm{mg}$ twice daily) for at least four months, $77 \%$ of patients with a duration of disease less than 10 years had a clinical response (at least 6\% improvement in SALT score) (35). Furthermore, three patients treated with ruxolitinib for myelofibrosis and another treated with barocitinib for CANDLE syndrome experienced near complete regrowth of hair within three to five months in the setting of moderate to severe alopecia areata (39)(40). Further support for a potential role for Janus kinase inhibitors in AA is the positive results of topical ruxolitinib and tofacitinib in mouse models (41) and a human randomised placebo-controlled case series (42). No topical formulation is currently available. The expert consensus group recommends involving a dermatologist with experience prescribing Janus kinase inhibitors in these cases.

A number of other systemic agents have also been reported in case reports or small uncontrolled retrospective case-series. These include simvastatin/ezetimibe (43), dapsone, mycophenolate mofetil, tacrolimus, sulfasalazine and psoralen photochemotherapy. Apart from the combination of simvastatin/ ezetimibe, which may work through inhibition of the JAK/stat pathway, the evidence for these agents is weak (44). While we are aware that many dermatologists have trialed these agents in AA for 3 to 6 months in patients who fail to respond to other systemic treatments, the expert consensus group does not recommend their use.

\section{Evaluating Treatment Response}

This article is protected by copyright. All rights reserved 
Clinical response should ideally be measured through history, assessment of hair shedding severity, serial photography, SALT score and quality of life measures including Dermatology Life Quality Index (DLQI) and Alopecia Areata Symptom Impact Scale (AASIS), comparing response at the most recent visit to baseline. A SALT50 denotes achieving 50\% improvement in SALT and is an acceptable goal for treatment success. Disease activity is commonly associated with increased hair shedding (stages 4-6) and a positive hair pull test, especially at the edge of existing patches of alopecia. The presence of exclamation mark hairs and black dots on dermoscopy are also indicators of active disease.

\section{Cessation of treatment}

For systemic glucocorticoids, treatment should be weaned over 3 months to reduce the risk of side effects known to be associated with long-term use.

For patients who have responded to steroid-sparing agents, the treatment should ideally be continued for 3-6 months post complete remission to minimise the risk of relapse. The dose can be gradually tapered during this time.

Minor relapses may occur as the dose is weaned. Options to treat minor relapses include dose escalation, the use of intralesional triamcinolone or short-term use of systemic glucocorticoids.

Patients who develop a major relapse (i.e. multiple additional new patches) while on adequate doses of systemic therapy should stop the current treatment and consider whether to commence an alternate steroid sparing agent. Additional treatment for a major relapse may include the use of intralesional triamcinolone and/or short-term use of systemic glucocorticoids.

Treatment may also need to be ceased in patients who develop significant toxicity secondary to these systemic agents. 
Progression to steroid sparing agents is not mandatory and many patients will elect to discontinue treatment and let the AA take its course. These patients can access expert advice on wigs through specialist centres and the Australia Alopecia Areata Foundation (www.aaaf.com.au).

\section{Conclusion}

This consensus statement draws upon the clinical expertise of dermatologists experienced in the treatment of AA. The advice is supported by hair biologists familiar with the aetiology, pathogenesis, and current scientific literature on the biology of hair growth in general and AA in particular. These guidelines, summarised in Figure 1, provide an Australian framework for assessment and treatment of AA.

\section{References}

1. Safavi KHMS, Suman VJ, Moshell AN, Melton $\amalg$, 3rd. Incidence of alopecia areata in Olmsted County, Minnesota, 1975 through 1989. Mayo Clinic proceedings Mayo Clinic. 1995;70(7):628-33.

2. Madani S SJ. Alopecia areata update. Journal of the American Academy of Dermatology. 2000;42(4):549-66.

3. Ikeda T. A New Classification of Alopecia Areata. Dermatology. 1965;131(6):421-45.

4. Delamere FM SM, Dobbins HM, Leonardi-Bee J. Interventions for alopecia areata. Cochrane Database Syst Rev. 2008;16(2):CD004413.

5. Lai V CG, Sinclair R. . Systemic treatments for alopecia areata: a systematic review. Australasian Journal of Dermatology. 2018; In Press.

6. Alkhalifah A AA, Wang E, McElwee KJ, Shapiro J. Alopecia areata update: part I. Clinical picture, histopathology, and pathogenesis. J Am Acad Dermatol. 2010;62(2):177-88. This article is protected by copyright. All rights reserved 
7. Jang YH, Hong N-S, Moon SY, Eun DH, Lee WK, Chi SG, et al. Long-term prognosis of alopecia totalis and alopecia universalis: A Longitudinal study with more than 10 years of follow-up: Better than reported. Dermatology. 2017;233(2-3):250-6.

8. Tosti A BS, lorizzo M. Alopecia areata: a long term followup study of 191 patients. J Am Acad Dermatol. 2006;55(1):438-41.

9. Ruiz-Doblado S CA, García-Hernández MJ. Alopecia areata: psychiatric comorbidity and adjustment to illness. International journal of dermatology. 2003;42(6):434-7.

10. KooJY SW, Hallman CP, Edwards JE. Alopecia areata and increased prevalence of psychiatric disorders. International journal of dermatology. 1994;33(12):849-50.

11. A G. Comorbidity of psychiatric disorders in children and adolescents with alopecia areata in a child and adolescent psychiatry clinical sample. International journal of dermatology. 2008 Nov 1;;47(11):1118-20.

12. Shapiro J MS. Alopecia areata: diagnosis and management. International journal of dermatology. 1999;38(S1):19-24.

13. MacDonald Hull SP WM, Hutchinson PE, Sladden M, Messenger AG. Guidelines for the management of alopecia areata. British Journal of Dermatology. 2003;149(4):692-9.

14. Price V. Double-blind, placebo-controlled evaluation of topical minoxidil in extensive alopecia areata. Journal of the american academy of dermatology [Internet]. 1987; 16(3 Pt 2):[730-6 pp.]. Available from:

http://onlinelibrary.wiley.com/o/cochrane/clcentral/articles/193/CN-00047193/frame.html. 15. Tosti A, Morelli R, Bardazzi F, Peluso Anna M. Prevalence of Nail Abnormalities in Children With Alopecia Areata. Pediatric Dermatology. 2008;11(2):112-5.

16. Gandhi V, Baruah M, Bhattacharaya S. Nail changes in alopecia areata: Incidence and pattern. Indian Journal of Dermatology, Venereology, and Leprology. 2003;69(2):114-5.

17. Tosti A, Fanti PA, Morelli R, Bardazzi F. Trachyonychia associated with alopecia areata: a clinical and pathologic study. Journal of the American Academy of Dermatology. 1991;25(2):266-70.

18. Thomas EA, Kadyan RS. ALOPECIA AREATA AND AUTOIMMUNITY: A CLINICAL STUDY. Indian Journal of Dermatology. 2008;53(2):70-4.

19. van der Steen PHM, van Baar HMJ, Happle R, Boezeman JBM, Perret CM. Prognostic factors in the treatment of alopecia areata with diphenylcyclopropenone. Journal of the American Academy of Dermatology.24(2):227-30.

This article is protected by copyright. All rights reserved 
20. Walker SA, Rothman S. Alopecia Areata1: A Statistical Study and Consideration of Endocrine Influences. Journal of Investigative Dermatology. 1950;14(6):403-13.

21. Waisman M, Kepler EJ. Alopecia areata: An appraisal of endocrine factors in its causation. Journal of the American Medical Association. 1941;116(18):2004-6.

22. Tan $E$, Tay $Y K$, Goh CL, Chin Giam Y. The pattern and profile of alopecia areata in Singapore-a study of 219 Asians. International journal of dermatology. 2002;41(11):748-53.

23. Petukhova L, Duvic M, Hordinsky M, Norris D, Price V, Shimomura Y, et al. Genomewide association study in alopecia areata implicates both innate and adaptive immunity. Nature. 2010;466:113.

24. Betz RC, Petukhova L, Ripke S, Huang H, Menelaou A, Redler S, et al. Genome-wide meta-analysis in alopecia areata resolves HLA associations and reveals two new susceptibility loci. Nature communications. 2015;6:5966-.

25. Du Vivier A, Munro DD. Alopecia areata, autoimmunity, and Down's syndrome. British Medical Journal. 1975;1(5951):191-2.

26. Alves R, Ferrando J. Alopecia areata and Down's syndrome. International Medical Review on Down Syndrome. 2011;15(3):34-6.

27. Xavier AC, Ge Y, Taub JW. Down Syndrome and Malignancies: A Unique Clinical Relationship: A Paper from the 2008 William Beaumont Hospital Symposium on Molecular Pathology. The Journal of Molecular Diagnostics : JMD. 2009;11(5):371-80.

28. Ustuner $\mathrm{P}$, Balevi A, Ozdemir M. Best dilution of the best corticosteroid for intralesional injection in the treatment of localized alopecia areata in adults. Journal of dermatological treatment [Internet]. 2017; 28(8):[753-61 pp.]. Available from:

http://onlinelibrary.wiley.com/o/cochrane/clcentral/articles/518/CN-01442518/frame.html. 29. Chu TW, AlJasser M, Alharbi A, Abahussein O, McElwee K, Shapiro J. Benefit of different concentrations of intralesional triamcinolone acetonide in alopecia areata: An intrasubject pilot study. Journal of the American Academy of Dermatology. 2015;73(2):33840.

30. Ganjoo S, Thappa D. Dermoscopic evaluation of therapeutic response to an intralesional corticosteroid in the treatment of alopecia areata. Indian Journal of Dermatology, Venereology, and Leprology. 2013;79(3):408-17. 
31. Kurosawa M, Nakagawa S, Mizuashi M, Sasaki Y, Kawamura M, Saito M, et al. A Comparison of the Efficacy, Relapse Rate and Side Effects among Three Modalities of Systemic Corticosteroid Therapy for Alopecia Areata. Dermatology. 2006;212(4):361-5.

32. Dehghan M, Alborzi A, Shahini N. Comparison of oral prednisolone pulse therapy with intravenous methylprednisolone pulse therapy in severe alopecia areata. Journal of Pakistan Association of Dermatologists. 2013;23.

33. Kar B, Handa S, Dogra S, Kumar B. Placebo-controlled oral pulse prednisolone therapy in alopecia areata. Journal of the american academy of dermatology [Internet]. 2005; 52(2):[287-90 pp.]. Available from:

http://onlinelibrary.wiley.com/o/cochrane/clcentral/articles/985/CN-00520985/frame.html. 34. Hubiche T, Léauté-Labrèze C, Taïeb A, Boralevi F. Poor long term outcome of severe alopecia areata in children treated with high dose pulse corticosteroid therapy. British Journal of Dermatology. 2008;158(5):1136-7.

35. Liu LY, Craiglow BG, Dai F, King BA. Tofacitinib for the treatment of severe alopecia areata and variants: a study of 90 patients. Journal of the American Academy of Dermatology. 2017;76(1):22-8.

36. Craiglow BG, Liu LY, King BA. Tofacitinib for the treatment of alopecia areata and variants in adolescents. Journal of the American Academy of Dermatology. 2017;76(1):2932.

37. Gupta A, Carviel J, Abramovits W. Efficacy of tofacitinib in treatment of alopecia universalis in two patients. Journal of the European Academy of Dermatology and Venereology. 2016;30(8):1373-8.

38. Ibrahim O, Bayart CB, Hogan S, Piliang M, Bergfeld WF. Treatment of alopecia areata with tofacitinib. JAMA dermatology. 2017;153(6):600-2.

39. Messenger A, McKillop J, Farrant P, McDonagh A, Sladden M. British Association of Dermatologists' guidelines for the management of alopecia areata 2012. British journal of dermatology. 2012;166(5):916-26.

40. Jabbari A, Dai Z, Xing L, Cerise JE, Ramot $Y$, Berkun $Y$, et al. Reversal of alopecia areata following treatment with the JAK1/2 inhibitor baricitinib. EBioMedicine. $2015 ; 2(4): 351-5$

This article is protected by copyright. All rights reserved 
41. Xing L, Dai Z, Jabbari A, Cerise JE, Higgins CA, Gong W, et al. Alopecia areata is driven by cytotoxic T lymphocytes and is reversed by JAK inhibition. Nature medicine. 2014;20(9):1043.

42. Bokhari LSR. Treatment of alopecia universalis with topical Janus Kinase inhibitors a double blind, placebo and active controlled pilot study. International Journal of dermatology. 2018; In press.

43. Lattouf C, Jimenez JJ, Tosti A, Miteva M, Wikramanayake TC, Kittles C, et al. Treatment of alopecia areata with simvastatin/ezetimibe. Journal of the American Academy of Dermatology. 2015;72(2):359-61.

44. DelCanto G, Bayer A, Kindelan CC, Mendez A, Jimenez J. 081 Simvastatin reduces autoimmune alopecia through direct action on T lymphocytes. Journal of Investigative Dermatology. 2018;138(5):S14.

Figure 1: Treatment algorithm for systemic treatment of alopecia areata

Tables:

Table 1. Prognosis alopecia areata based on study of 1989 patients seen in Kyoto, Japan between 1947 and 1963 (3)

\begin{tabular}{|l|l|l|l|}
\hline Clinical presentation & Risk of & Risk of & Risk of \\
& spontaneous & developing & developing \\
& regrowth & Chronic AA & AT or AU \\
\hline Solitary Stable Patch of AA $\leq 6$ months & $87 \%$ & $13 \%$ & $6 \%$ \\
duration & & & \\
\hline Solitary Stable Patch of AA $\geq 6$ months but & $35 \%$ & $65 \%$ & $30 \%$ \\
\hline
\end{tabular}

This article is protected by copyright. All rights reserved 


\begin{tabular}{|l|l|l|l|}
\hline$\leq 12$ months & & & \\
\hline Solitary Stable Patch of AA $\geq 12$ months & 0 & $100 \%$ & $45 \%$ \\
\hline Muration & $65 \%$ & $35 \%$ & $15 \%$ \\
\hline Children aged $0-6$ years at initial presentation & 0 & & \\
\hline
\end{tabular}

Table 2. Proportion of patients with Chronic AA in 2012 who had subsequently developed AT or AU by 2018.

\begin{tabular}{|l|c|}
\hline Number of patients with chronic AA who received & 68 \\
systemic treatment and who have been followed & \\
for a minimum of 5 years year. & $1: 2.6$ \\
\hline Male to female ratio & 3 \\
\hline Number of children aged 6 or under at onset of & \\
\hline
\end{tabular}




\begin{tabular}{|l|c|}
\hline Number of children aged 6 or under at onset of & 1 \\
\hline Number of patients with chronic AA who & $12(17.6 \%)$ \\
progressed to AT/AU while on treatment & $56(82.3 \%)$ \\
\hline $\begin{array}{l}\text { Number of patients who continue to have chronic } \\
\text { multi-focal patchy AA but who have not }\end{array}$ & \\
progressed to AT or AU & \\
\hline Number of patients who developed AT/AU while & \\
on treatment and who subsequently regrew either & \\
spontaneously or with subsequent systemic & \\
treatment
\end{tabular}

Table 3. Systemic therapy continuation rates at 12 months

\begin{tabular}{|l|l|l|l|l|}
\hline Agent & Non- & Responders $(\mathrm{n} / \mathrm{n})$ & Proportion of & Average daily dose \\
& Responders & $(\%)^{*}$ & responders also & of concurrent \\
& $(\mathrm{n} / \mathrm{n})(\%)^{*}$ & & using concurrent & prednisolone $(\mathrm{mg})$ \\
& & prednisolone $(\mathrm{n} / \mathrm{n})$ & \\
& & $(\%)$ & \\
\hline
\end{tabular}




\begin{tabular}{|l|l|l|l|l|}
\hline Ciclosporin & $9 / 20(45 \%)$ & $11 / 20(55 \%)$ & $7 / 11(64 \%)$ & 6.4 \\
\hline Methotrexate & $11 / 20(55 \%)$ & $9 / 20(45 \%)$ & $5 / 9(56 \%)$ & 4.8 \\
\hline Azathioprine & $7 / 20(35 \%)$ & $13 / 20(65 \%)$ & $5 / 13(38 \%)$ & 5.6 \\
\hline
\end{tabular}

*Responders are defined as patients who have either continued to take the medication for 12 months or longer or who have stopped the medication due to complete remission. Nonresponders are defined as patients who stopped the medication prior to 12 months either due to side-effects or lack of efficacy (including relapse while on treatment).

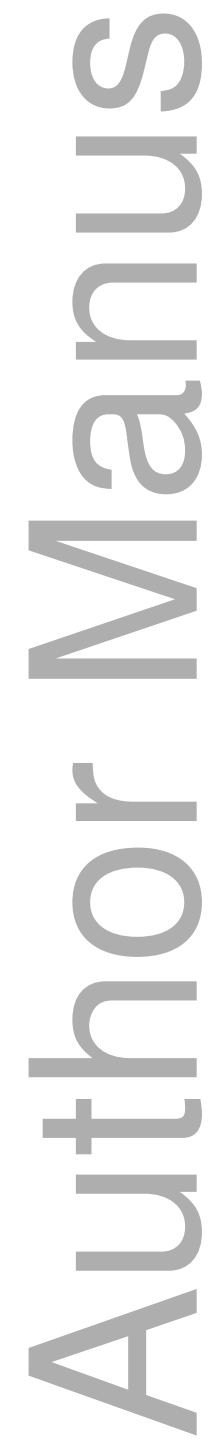




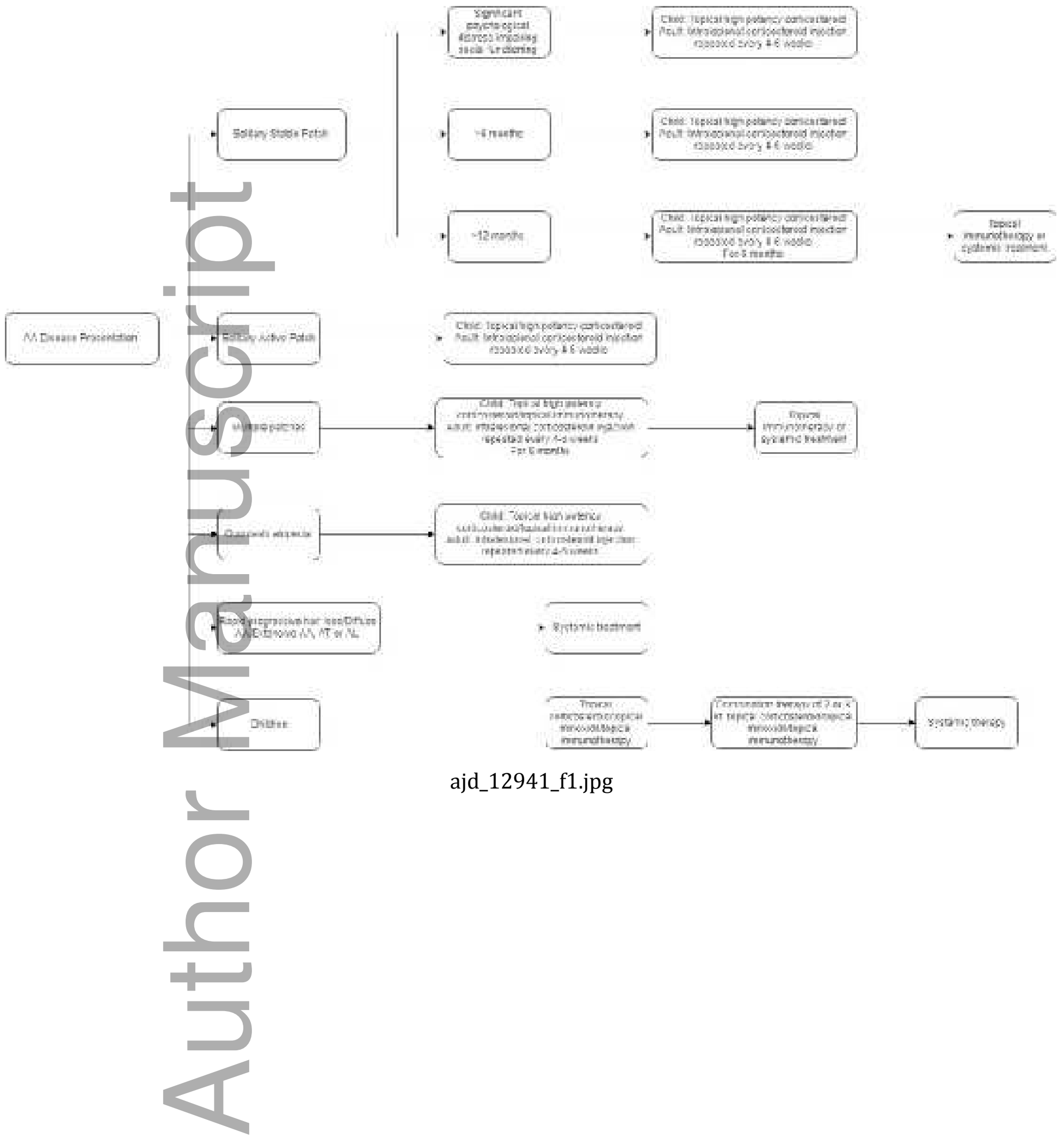




\section{University Library}

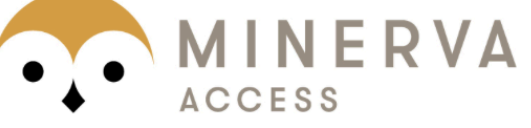

A gateway to Melbourne's research publications

Minerva Access is the Institutional Repository of The University of Melbourne

\section{Author/s:}

Cranwel, WC;Lai, VWY;Photiou, L;Meah, N;Wall, D;Rathnayake, D;Joseph, S;Chitreddy,

V;Gunatheesan, S;Sindhu, K;Sharm, P;Green, J;Eisman, S;Yip, L;Jones, L;Sinclair, R

Title:

Treatment of alopecia areata: An Australian expert consensus statement

Date:

2019-05-01

\section{Citation:}

Cranwel, W. C., Lai, V. W. Y., Photiou, L., Meah, N., Wall, D., Rathnayake, D., Joseph, S., Chitreddy, V., Gunatheesan, S., Sindhu, K., Sharm, P., Green, J., Eisman, S., Yip, L., Jones, L. \& Sinclair, R. (2019). Treatment of alopecia areata: An Australian expert consensus statement. AUSTRALASIAN JOURNAL OF DERMATOLOGY, 60 (2), pp.163-170. https:// doi.org/10.1111/ajd.12941.

Persistent Link:

http://hdl.handle.net/11343/284773 